\title{
La interpretación easterliniana de la fecundidad. Consenso y polémica en torno a la obra del autor*
}

\author{
Carmen Rodríguez Sumaza
}

Universidad de Valladolid. Facultad de Ciencias Económicas. Departamento de Sociología Avda. Valle Esgueva, 6. 47011 Valladolid

\section{Resumen}

El artículo tiene como objeto sintetizar la labor investigadora que el demógrafo y economista norteamericano Richard A. Easterlin desarrolla a lo largo de su obra. La relevancia de la contribución del autor a la comprensión de las decisiones en materia de fecundidad ha sido y es internacionalmente reconocida, pero también fuente de vivas polémicas. Sus principales tesis y los más destacados trabajos de validación de las mismas que han ido apareciendo y que han contribuido con un denso debate a la profundización en el tema son aquí analizados.

Abstract. The Easterlin's interpretation of the fertility. Consensus and controversy about yours works

The aim of the paper is to summarize the research carried out by the Northamerican demographer and economist Richard A. Easterlin in relation to fertility and economic status. The importance of the contribution of this author in the understanding of the decisions about fertility behavior has been internationally recognized but at the same tiem it has been the source of a lively debate. The paper also reviews the most outstanding research to validate Easterlin's propositions.

\section{Sumario}

1. Introducción determinantes de los niveles

2. La perspectiva socioeconómica en el de fecundidad: la hipótesis de Easterlin

estudio de la fecundidad. Una propuesta sintetizadora de las explicaciones sociológica y económica

3. El status económico relativo y el tamaño relativo de la cohorte como

4. El análisis de la evidencia: los intentos de verificación de la hipótesis de Easterlin

5. Conclusiones

Bibliografía

* Quiero expresar mi agradecimiento a D. Juan Salcedo Martínez por toda la ayuda y orientación recibida. Buena parte de esta investigación fue realizada durante mi estancia como profesor visitante en el Center for Studies in Demography and Ecology del Departamento de Sociologia de la Universidad de Washington, Seattle (Estados Unidos), gracias a la financiación de la Universidad de Valladolid. Agradezco a los profesores Charles Hirschman, Diane Lye y William Lavely sus consejos y las discusiones mantenidas. 


\section{Introducción}

Tras la finalización del proceso de transición demográfica en el conjunto de los países más desarrollados, todas las previsiones apuntaban a la persistencia en el tiempo de unos niveles de fecundidad bajos y estables acordes con unas tasas de mortalidad también reducidas. Sin embargo, contra todo pronóstico, se asistió al alza generalizada del número medio de hijos por mujer, fenómeno que fue seguido a continuación de un nuevo cambio de tendencia con la caída brusca del citado indicador.

Cautivados por tan insólito comportamiento y conscientes de sus profundas repercusiones futuras, los analistas del momento concentraron sus esfuerzos en encontrar una explicación a los hechos. Sociólogos y economistas entre otros ${ }^{1}$ desarrollan a partir de la décadla de los sesenta importantes líneas de investigación que constituyen inicialmente verdaderas explicaciones alternativas. En el intento por localizar a los factores responsables de las nuevas trayectorias de la fecundidad se van a barajar hipótesis de muy distinta naturaleza, y pronto nace una rivalidad entre los profesionales de ambas disciplinas sociales. En un principio, el producto del análisis de ambos grupos de investigadores era claramente diferenciable, y nada parecía sugerir que hubiera algún interés por parte de unos u otros por conocer las aportaciones que desde el otro enfoque iban apareciendo ${ }^{2}$. En este contexto se desarrolla la obra del economista y demógrafo norteamericano Richard A. Easterlin, que persigue una integración de los elementos explicativos defendidos desde los ámbitos de la sociología y la economía.

El presente artículo trata en primer lugar de destacar la oportunidad histórica de la aportación del autor, en un momento crítico en que los distintos científicos sociales se afanaban por buscar explicaciones en el marco estricto de sus disciplinas, ignorando conscientemente en la mayoría de los casos otros puntos de vista, otros elementos de análisis complementarios.

Se destacan a continuación algunos de los argumentos de mayor interés defendidos por el autor a lo largo de su obra y, finalmente, se hace un repaso de los numerosos intentos por parte de otros autores de validar empíricamente la hipótesis formulada por Easterlin. Se pretende con todo ello ofrecer una visión global de lo que ha supuesto la aportación del autor al desarrollo de la teoría demográfica y de las repercusiones científicas de la misma.

1. El estudio de los determinantes de la fecundidad se ha visto también enriquecido con aportaciones procedentes de otras ciencias como son la psicología, la antropología, la medicina, la biología, etc.

2. La revisión de la literatura reciente sobre fecundidad de economistas y sociólogos resulta inicialmente sorprendente por su fragmentación, pero a la vez genera en el lector una cierta sensación de frustración por la competencia existente entre las diferentes alternativas. 
2. La perspectiva socioeconómica en el estudio de la fecundidad. Una propuesta sintetizadora de las explicaciones sociológica y económica

Desde el ámbito de la sociología, los estudios acerca del comportamiento en materia de fecundidad han estado tradicionalmente orientados a explicar el porqué de las diferencias en las tasas de fecundidad entre las distintas sociedades, así como las diferencias existentes entre las tasas de fecundidad de las parejas dentro de una misma sociedad. Para ello, han desarrollado un marco de trabajo que subraya la importancia de los factores culturales y sociales en la toma de decisiones por parte de los individuos en materia de fecundidad (Jones, 1982).

En el intento de sistematizar el efecto de los elementos sociales sobre los niveles de fecundidad destaca la aportación teórica pionera de Davis y Blake (1956). El modelo sociológico para el análisis de los niveles de fecundidad propuesto por ambos señala la existencia de un conjunto de variables denominadas "intermedias" (por ejemplo: la edad en que se contrae matrimonio, la práctica de contracepción, etc.) que materializan la influencia que las normas y los hábitos culturales y sociales tienen sobre la fecundidad. Las características del entorno de los individuos, que son los determinantes indirectos del nivel de fecundidad, afectan al nivel de las variables intermedias modificando las normas sociales, los valores vigentes en la sociedad (por ejemplo: la idea acerca de cuál es la edad más apropiada para el matrimonio). Las variables intermedias son el factor clave en este esquema analítico, pues sólo a través de ellas las condiciones culturales pueden afectar a la fecundidad. La propuesta de estos autores se presenta esquematizada en la figura 1.

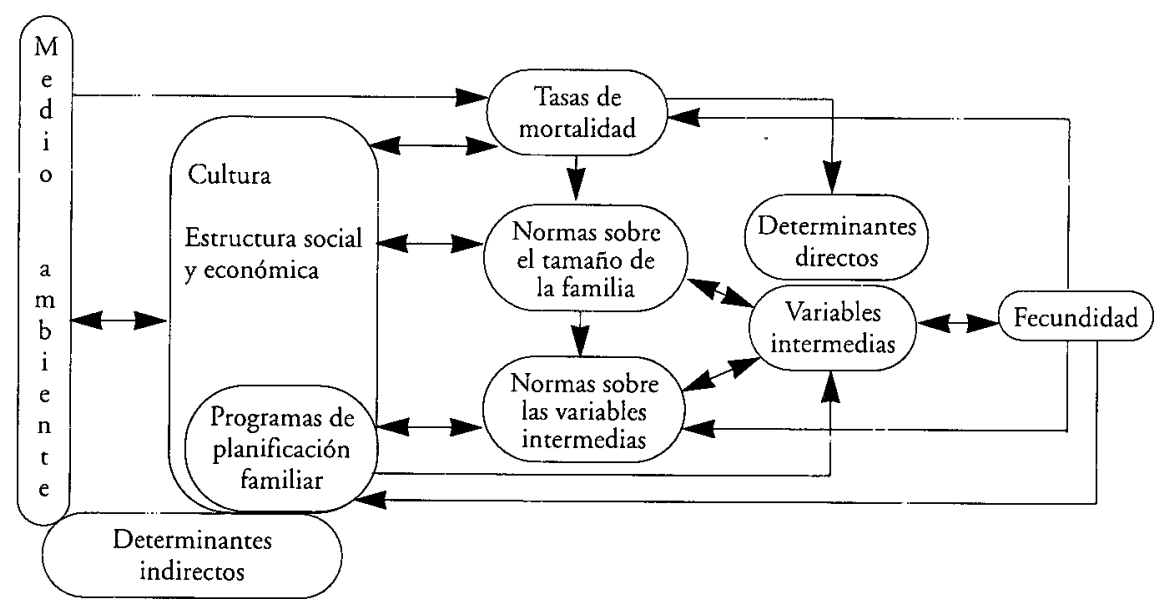

Figura 1. Determinantes directos e indirectos de la fecundidad. Fuente: Freedman, R. (1975), p. 15 y elaboración propia. 
La determinación y cuantificación de estas variables y de su relación con los niveles de fecundidad ha sido una de las tareas que más ampliamente ha ocupado a los sociólogos de la población interesados en este tema ${ }^{3}$. Otros autores han orientado su trabajo al estudio histórico del peso relativo de este tipo de factores. Así, Bourgeois-Pichat (1967) y Wrigley (1969) se centran en el análisis de la diferente naturaleza de los condicionantes de la fecundidad en las sociedades preindustriales y en las sociedades modernas, donde ya ha finalizado la transición demográfica.

Siguiendo a Easterlin (1971b), se puede afirmar que la aportación más significativa de la sociología al análisis del comportamiento en materia de fecundidad ha sido su enfoque del tema "pos: el lado de la producción", sintetizando así la idea de que, en general, los modelos existentes intentan básicamente explicar el número acumulado de hijos vivos por familia ${ }^{4}$. Esta variable se hace depender de la fecundidad natural (número acumulado de nacidos vivos si no hubiera control voluntario de la fecundidad) y de una medida sintética de la práctica y la eficiencia de la regulación voluntaria de la fecundidad. La fecundidad natural, a su vez, se ve afectada tanto por factores biológicos y psicológicos como por factores culturales, y sus principales componentes son la frecuencia de las relaciones sexuales, la fertilidad y la mortalidad fetal involuntaria.

El análisis sociológico de los determinantes de la regulación de la fecundidad abarca cuestiones relacionadas con cuáles son los motivos que llevan a las parejas a controlar el número de hijos y/o determinar su espaciamiento, cuáles son las actitudes o el grado de aceptación por parte de los individuos de las diferentes prácticas contraceptivas y qué grado de conocimiento y acceso tiene la población a este tipo de prácticas.

La principal corriente teórica que domina en la actualidad la explicación económica de la fecundidad surge en los años sesenta a partir del trabajo que Gary Becker (1960) presenta en el seno de una conferencia sobre el papel de los factores demográficos en el cambio económico en los países desarrollados 5 . En él Becker desarrolla la idea de que las variaciones de la fecundidad pueden ser entendidas en el mismo marco que los economistas usan para analizar la demanda de los bienes de larga duración.

La interpretación que hace Becker del comportamiento procreador de los individuos se sitúa en el contexto general de la teoría neoclásica del consumo $y$, en particular, su formulación se enmarca en el ámbito de la teoría microeconómica del consumidor. Su propuesta analítica, puesta a prueba inicialmente

3. Un serio intento de formalización de la influencia de las variables intermedias sobre la fecundidad lo constituye el trabajo de Bongaarts (1978), que luego aplica al estudio de los cambios en los niveles de fecundidad en Corea y Estados Unidos.

4. Esta es la variable endógena del modelo.

5. Una revisión histórica del interés de los economistas por los temas relacionados con la población, desde Malthus en adelante, se puede encontrar en Spengler (1966) o, de una manera más sintética, en Del Hoyo y García Ferrer (1988). 
en la universidades de Chicago y Columbia y en el National Bureau of Economic Research, recibe el nombre de la primera de estas instituciones y se conoce como la Escuela de Chicago.

Para Becker los hijos pueden ser considerados como "bienes de consumo", ya que, al igual que el resto de los bienes, tienen unos costes y proporcionan unos beneficios. Estos últimos se derivan de la "satisfacción" que reporta el consumo de todo bien, el «rendimiento» que proporcionan los hijos al" constituir un factor de producción en las familias tradicionales y la «seguridad» que suponen para los padres de cara al futuro (Del Hoyo y García Ferrer, 1988, p. 26).

Dadas las preferencias de una pareja por hijos en oposición a otros bienes alternativos, cada pareja elegirá una combinación de hijos y otros bienes que les proporcione la máxima utilidad. La demanda de hijos es, por tanto, el resultado del equilibrio entre las preferencias subjetivas de las familias y las restricciones que imponen los precios (de los bienes relacionados con los hijos y de los bienes alternativos) y la renta, de manera que se maximice la utilidad (la satisfacción subjetiva). La consideración de los hijos como bienes de consumo generó desde un principio amplia polémica. Entre las críticas más contundentes a esta idea destacan las desarrolladas por Blake (1968) y Berelson (1972) ${ }^{6}$.

Una de las contribuciones más importantes de Becker es la introducción del concepto de «calidad» de los hijos en el análisis de la relación entre los ingresos familiares y la demanda de hijos. Dicho concepto permite comprender el hecho de que incrementos en los niveles de renta de las familias no van necesariamente asociados a incrementos en la demanda de hijos ${ }^{7}$ : cuando aumenta la renta familiar, también aumentan los gastos en la educación de los hijos, y por tanto es perfectamente posible que el número de hijos permanezca constante a pesar de que los ingresos familiares sean superiores. Un aumento en la calidad de los hijos hace elevar el precio de los hijos adicionales, lo que frena su demanda. Por otra parte, si aumenta la renta familiar, crece con ello el coste del tiempo (sobre todo de la madre) dedicado al cuidado de los hijos, lo que también hace elevar el precio de éstos y por tanto disminuir su demanda.

6. Un ejemplo de la crítica que suscitó en su momento la aparición de los primeros trabajos que analizaban la fecundidad desde una óptica estrictamente económica lo constituye el artículo de Blake (1968), que se centra tanto en el análisis de los supuestos teóricos que subyacen en dichos modelos como en el estudio de aquellos elementos o factores que no son considerados. Más recientemente, Carrasco (1988) se plantea la duda acerca de la operatividad de los modelos microeconómicos para enfocar el tema. Según ella, dichos modelos son descriptivos más que explicativos, y se podrían obtener iguales resultados aplicando únicamente análisis econométricos, sin necesidad por tanto de suponer esquemas de comportamiento previos

7. Sin la introducción del concepto de "calidad" de los hijos, la evidencia empírica llevaba a la conclusión de que éstos eran bienes inferiores, que son por definición aquéllos cuyo consumo disminuye cuando aumentan los ingresos. Esto se conoce como la «paradoja de los bienes inferiores". 
Destacan también dentro de esta escuela las aportaciones de Mincer (1962 y 1963), con su esclarecedora profuncización en conceptos clave en la toma de decisiones de consumo por parte de las familias como son la renta permanente y el precio de los hijos.

En 1973 y 1974 otras dos publicaciones, dos números especiales de la revista Journal of Political Economy ${ }^{8}$, contienen un conjunto importante de artículos que no solamente consolidan el trabajo teórico aparecido hasta el momento en el ámbito de la econornía de la familia, sino que sofistican tanto sus argumentos como los procedimientos empíricos necesarios para validar éstos. Tal y como explican Bagozzi y Van Loo (1978, p. 199), si hasta ahora los autores de la denominada Escuela de Chicago habían estudiado la fecundidad (número de hijos) como el resultado de una decisión de consumo por parte de la familia; a partir de ahora, se va a estudiar la demanda de hijos también como el resultado de un "proceso de decisión en el que el tiempo y los bienes de mercado se combinan para producir más bienes». Con este nuevo enfoque se da una visión más realista del problema de la decisión de una familia en materia de fecundidad: los hijos no se adquieren ya "hechos", sino que el proceso de crianza y educación corresponde a la propia familia. El hijo, por tanto, no es sólo un producto biológico, sino un producto de la actividad de la familia. Otra de las cuestiones más importantes es que se desarrolla con mayor precisión cuál ha de ser la valoración del coste de oportunidad del tiempo de la madre. Esta renovada visión económica de la fecundidad pasa a denominarse la nueva economía de la familia?.

La denominada escuela de la Nueva Economía de la Familia ha ido introduciendo diferentes modificaciones ${ }^{10} \mathrm{y}$ desarrollando formulaciones cada vez más sofisticadas de la propuesta básica de Becker, lo que constituye en la actualidad una teoría del consumidor "más moderna» que permite hacer un análisis "más flexible y refinado» del comportamiento en materia de fecundidad (Kyriazis, 1987, p. 168) ${ }^{11}$.

En 1966 Richard A. Easterlin publica un modelo que difiere radicalmente de la idea contenida en la propuesta de $\mathrm{G}$. Becker al negar la validez de uno de sus pilares fundamentales: el supuesto de que los gustos o las preferencias de los sujetos permanecen constantes en el tiempo. La idea inicial de Easterlin va a tener su origen en los trabajos de autores como Leibenstein (1957) sobre el papel del status social de las familias y de la educación en la formación de las

8. Schultz, Teodore W. (ed.) (1973) y (1974). Ambos volúmenes son luego reeditados en Schultz, T.W. (1974).

9. La denominación original es «New Home Economics» o también «New Household Economics".

10. El artículo de Namboodiri (1972) es un claro ejemplo de los esfuerzos realizados para tratar de incorporar algunas de las críticas que suscitó el trabajo inicial de Becker (1960) y conseguir así una teoría más potente.

11. Una síntesis de las principales críticas a esta teoría se puede encontrar en Bagozzi y Van Loo (1978), Jones (1982) o en Kyriazis (1987). 
preferencias $^{12}$, Grauman (1960) sobre las perspectivas demográficas y sobre todo Kuznets (1930 y 1958) y su análisis de la relación entre el crecimiento de la demanda agregada y los ciclos a largo plazo en las series demográficas ${ }^{13} \mathrm{y}$ Abramovitz (1964) ${ }^{14}$.

En su artículo, Easterlin muestra cómo los movimientos en las tasas específicas de fecundidad por edad de las mujeres jóvenes han estado relacionadas positivamente a lo largo del tiempo con un índice que él denomina "renta relativa intergeneracional». Este ratio, uno de los conceptos clave de su teoría, mide la relación existente entre la renta actual de las parejas casadas jóvenes y el nivel de renta de que ellos disfrutaron cuando eran adolescentes en casa de sus padres.

¿Qué trascendencia tiene esta reformulación de la idea original de Becker? En primer lugar, Easterlin no se conforma con rechazar la idea tradicional de que los gustos o preferencias han de ser siempre tratados como algo inmutable, sino que propone un mecanismo que permite considerar las modificaciones en las preferencias de los individuos en función de su educación. Esto significa que se está introduciendo el concepto de socialización en la modelización económica, lo que supone un cambio cualitativo radical en relación con la teoría económica estándar. Los gustos pueden variar de una generación a otra según el contexto en que el individuo ha sido educado. Como señala Kyriazis (1987, p. 176): «en la medida en que se acepte que el medio familiar es un indicador de clase social, se puede decir que, en cierto sentido, Easterlin incluye esta variable en su modelo".

Por otra parte, al hacerse depender los niveles actuales de fecundidad de los «ingresos relativos» de las parejas, se está relativizando el papel de las condiciones económicas del momento y se está introduciendo una metodología intertemporal (intergeneracional) en el análisis de estos fenómenos. Para Easterlin el nivel de fecundidad está condicionado por la diferencia entre las condiciones de vida actuales de los individuos y las que ellos vivieron de adolescentes, es decir, las de sus padres.

Por último, y esto es lo que definitivamente va a desvincular la propuesta de Easterlin del bloque de estudios económicos de la fecundidad, su propuesta no se apoya en un marco teórico tan rígido como es el de la teoría rentaprecios de Becker, sino que está mucho más abierta a la inclusión de elementos sociológicos y psicológicos en las explicaciones de la fecundidad. Esto hace

12. Para Leibenstein los cambios económicos influyen en el status social de los individuos y en sus gustos. La fecundidad de la pareja se determina en función de la renta relativa del marido, es decir, en base a su grupo de referencia socioeconómico. Si aumenta la renta familiar hay un cambio de status que hace que aumente el coste de criar a un hijo y por tanto no necesariamente aumenta la demanda de hijos. El tamaño de la familia se ve pues determinado por el deseo de ascender en la escala social.

13. El economista y Premio Nobel Simon Kuznets estudia cómo en Estados Unidos desde 1890 a 1955 las fluctuaciones de la inmigración y de las tasas de natalidad son el resultado de las variaciones en el crecimiento económico y en la formación del capital.

14. En el prefacio, Easterlin (1968a) reconoce su deuda con algunos de estos autores, así como con muchos de los trabajos desarrollados por el National Bureau of Economic Research. 


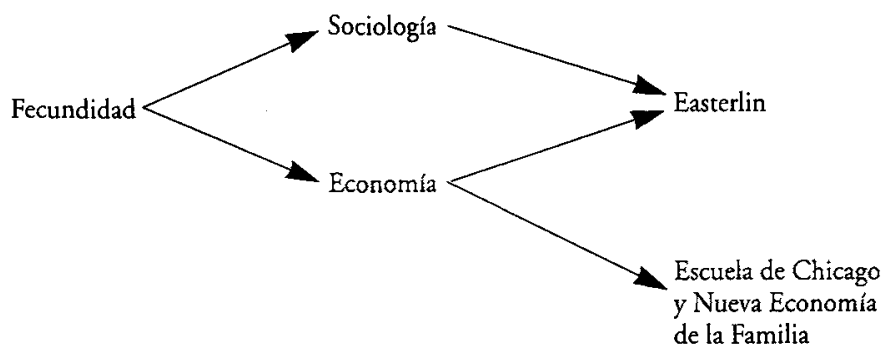

Figura 2.

que la potencialidad explicativa de su propuesta sea mayor, pero tiene como contrapartida una mayor dificultad a la hora de verificar su validez.

A partir de la publicación de los citados artículos de Becker y Easterlin va a quedar claramente delimitada la diferencia entre las explicaciones del comportamiento en materia de fecundidad basadas en la pura teoría económica y aquéllas que se apoyan para ello en elementos económicos y sociológicos. Algunos autores como Sanderson (1976, p. 470) han llegado a hablar de "cisma" para referirse a la escisión en dos grupos de economistas provocada por esta nueva interpretación. La escuela de la Nueva Economía de la Familia se caracteriza por poner el acento en el coste de los hijos como factor explicativo de la fecundidad. A diferencia de ésta, la constituida por Easterlin y sus seguidores, a través del concepto de ingresos relativos, se centra en la importancia de las aspiraciones materiales.

Quizás frente a esta clara dicotomía en el ámbito de los análisis económicos pueda parecer que las aportaciones de los sociólogos presentan una gran homogeneidad. Lejos de ser esto cierto, los trabajos de éstos son mucho menos uniformes entre sí a la hora de estudiar el tema, pero sin embargo hay un mayor consenso en la literatura sociológica acerca del tratamiento de las cuestiones a considerar.

La teoría que desarrolla Easterlin, que permite conciliar elementos propios del análisis económico con elementos sociológicos ${ }^{15}$, crea un conflicto en el seno de los economistas, al no haber un acuerdo unánime acerca de la universalidad de su planteamiento. La figura 2 representa la escisión que se produce en el ámbito de la economía en relación con el enfoque a adoptar en los análisis del comportamiento en materia de fecundidad y cómo la obra de Easterlin sintetiza o contiene elementos tanto económicos como sociológicos.

Easterlin vive muy de cerca el comentado conflicto entre economistas y sociólogos en su intento de explicación del comportamiento de los individuos

15. El propio Easterlin (1971b, p. 59) explica que su trabajo representa un umatrimonio entre iguales", en el sentido de que combina perfectamente tanto la ueconomía como la sociología de la fecundidad". 
en materia de fecundidad. Para él, el tradicional desinterés de los sociólogos por la contribución de la teoría económica al estudio de la fecundidad es el resultado de ver las propuestas de los economistas como una descripción simple acerca del efecto de la renta sobre el comportamiento de los individuos, ignorando el papel de los factores considerados clave por la sociología: religión, educación, procedencia de un entorno rural, etc. (Easterlin, 1969).

Su condición de economista le hace buen conocedor de la propuesta teórica desarrollada en el marco de la teoría económica convencional, pero su condición de científico social le lleva a la necesidad de hacer compatibles las visiones alternativas de las disciplinas económica y sociológica. Para Easterlin la clave está en diseñar o definir un marco teórico que permita considerar conjuntamente las variables clave empleadas por economistas, por un lado, y por sociólogos, por otro, ya que según él las contribuciones de ambos no son incompatibles. Su objetivo, por tanto, va a ser desarrollar una propuesta que comprenda todas las variables consideradas en ambas disciplinas y, en este contexto, clarificar las interrelaciones entre todas ellas.

Sin embargo, el deseo final de Easterlin es no sólo desarrollar un marco teórico amplio que permita incluir conceptos de economistas, sociólogos, demógrafos y otros científicos sobre la fecundidad, sino que también éste sea válido para explicar niveles pasados y actuales de fecundidad, que sirva para predecir, para explicar tendencias, oscilaciones y diferencias en los niveles de fecundidad históricos y fenómenos tales como la transición demográfica y las variaciones en la fecundidad premoderna (Easterlin, 1975a). Toda su fecunda producción científica va a estar orientada por tanto a formalizar y sistematizar su propuesta teórica y a desarrollar las posibilidades teóricas y empíricas de aplicación de la misma, con especial atención a la evolución de los niveles de fecundidad en el caso de los Estados Unidos.

\section{El status económico relativo y el tamaño relativo de la cohorte como determinantes de los niveles de fecundidad: la hipótesis de Easterlin}

Una visión de conjunto de la obra de Easterlin permite diferenciar dos niveles de análisis que se combinan y se coordinan continuamente. Por un lado estaría la perspectiva individual -especialmente evidente en Easterlin (1969), (1971b) y (1975a) - , que se centra en el estudio de los factores que afectan a las decisiones de las familias y a su comportamiento en materia de fecundidad. Es habitual en este apartado el abundante uso de elementos y conceptos tanto microeconómicos como demográficos para constituir lo que él denomina un modelo socioeconómico de determinación de la fecundidad, conocido también como teoría socioeconómica de la fecundidad.

Por otro lado, y desde una óptica agregada, Easterlin pretende explicar las variaciones de la fecundidad en el tiempo de aquellas sociedades en que ya ha finalizado la transición demográfica, pues sólo en éstas la evolución de la natalidad es independiente de la mortalidad y se ajusta a otros factores como son el status económico relativo de los jóvenes y el número relativo de éstos. La 
interpretación que se desarrolla a este nivel se conoce como la hipótesis de la renta relativa, o hipótesis de Easterlin. En este nivel de estudio se centrará a partir de ahora el presente trabajo ${ }^{16}$.

Easterlin, basándose en la experiencia norteamericana, explica las variaciones de la fecundidad como una consecuencia de las variaciones en el status económico relativo de las familias. Esto significa que, ceteris paribus, la tasa global de fecundidad de una cohorte tiende a variar directamente con el éxito económico y social de los individuos de esa cohorte. Cuanto más recursos tengan los individuos para satisfacer sus necesidades materiales - generadas en los años de adolescencia en el domicilio familiar-, con más facilidad formarán una familia y tendrán hijos.

El concepto de status económico relativo (SER) ha sido definido como uno de los aspectos más provocativos de la teoría de Richard A. Easterlin en relación con las oscilaciones a largo plazo en el crecimiento económico y la fecundidad (Oppenheimer, 1976, p. 443). Dicho concepto comprende dos variables: una de ellas representa el volumen de recursos económicos disponibles por las unidades familiares; la otra recoge la cuestión de sus aspiraciones materiales. Esta relación entre ganancias y aspiraciones se puede definir a través del siguiente ratio:

$$
\text { Status económico relativo }(\mathrm{SER})=\frac{\text { ingresos potenciales de la pareja }}{\text { aspiraciones materiales }}
$$

La tensión que surge entre las variables indicadas puede tener importantes efectos directos sobre la fecundidad, ya que, por ejemplo, parejas con altos niveles de ingresos pueden encontrar éstos insuficientes si sus aspiraciones de nivel de vida son muy elevadas, y como consecuencia de ello limitar el tamaño de familia para estirar los recursos disponibles (Freedman, 1976). Si la renta potencial de los individuos es alta en relación con sus aspiraciones materiales, será más fácil financiar éstas y por tanto habrá mayores recursos para formar una familia y tener hijos. Las decisiones de formación de una familia (matrimonio e hijos) se hacen por tanto depender de los ingresos relativos de las parejas.

Esta explicación podría ser denominada como explicación económica de la fecundidad, o versión económica de la hipótesis de Easterlin, porque los factores que determinan la fecundidad son básicamente económicos (tasas de paro, nivel salarial del cabeza de familia, etc.), a pesar de que los factores sociales estén también implicados en la determinación de las aspiraciones materiales.

Surge entonces el problema de cómo proceder a medir el status económico relativo. En un primer momento, Easterlin (1966, 1968a y 1973) propone medir el status económico relativo comparando la posición económica actual de los jóvenes trabajadores con la posición económica que en el pasado tuvie-

16. Una profundización en los distintos argumentos y variables empleados en ambos niveles de análisis, así como un análisis crítico de los mismos, se puede encontrar en Rodríguez Sumaza (1994). 
ron sus padres, siendo esta última variable representativa de las aspiraciones materiales desarrolladas por los jóvenes de hoy en el domicilio familiar. A mayores ingresos de los jóvenes en relación con los ingresos de sus padres, mayor capacidad económica y por tanto mayor facilidad para crear una familia y tener hijos.

De esta manera, el ratio inicial (SER) pasa a ser ahora una comparación de la experiencia económica de la nueva generación con relación a la generación precedente unos años antes, es decir, una medida de la renta relativa de los jóvenes (RR). Esto se podría expresar en los nuevos términos del siguiente modo:

$$
\text { Renta relativa }(R R)=\frac{\text { nivel ingresos actuales jóvenes varones }}{\text { nivel ingr. pasados padres jóvenes varones }}
$$

La segunda de las propuestas de Easterlin es medir los ingresos potenciales de la pareja (del varón) a través de su experiencia actual en el mercado de trabajo, ya que según él la valoración de las ganancias a lo largo de la vida está basada en gran parte en el actual nivel económico de los individuos y en su situación laboral actual (Easterlin, 1987a, p. 42).

En este caso se trataría de comparar la tasa de desempleo de los varones jóvenes o su experiencia laboral con la de sus padres varios ańos antes. Si la tasa de desempleo de los varones jóvenes es menor que la tasa de desempleo de sus padres cuando éstos eran jóvenes, eso significa que ha habido una mejora en su status económico, lo que facilita la financiación de sus aspiraciones materiales y la formación de familias. El nuevo indicador, que se convierte ahora en una medida del desempleo relativo (DR), se puede representar de la siguiente manera:

Desempleo relativo $(\mathrm{DR})=$

tasa desempleo pasada padres varones jóvenes

tasa desempleo actual varones jóvenes

A partir de 1976, Easterlin introduce una nueva variable explicativa en su modelo que hará que su explicación se torne eminentemente demográfica (Easterlin y Condran, 1976). Esta variable es la estructura por edad de la población. La estructura por edad de la población, bajo las condiciones características del período posterior a la Segunda Guerra Mundial en Estados Unidos (alto y sostenido crecimiento de la demanda agregada y restricciones a la inmigración), tiene un efecto importante sobre la posición económica relativa de los jóvenes varones: el nivel de ingresos relativos de los jóvenes varones, aquéllos de los que depende básicamente la formación de las nuevas familias, está determinado por el peso de los jóvenes trabajadores en relación con los trabajadores de mayor edad. La estructura por edad de la población en edad de trabajar determina la renta relativa de los varones jóvenes y ésta a su vez la fecundidad.

En esta nueva versión, la fortuna económica y social de una cohorte tiende a variar inversamente con su tamaño relativo. Se pone en marcha entonces 
lo que se conoce como el mecanismo de la superpoblación: si una cohorte es grande, sus miembros encontrarán a lo largo de su vida menores facilidades, mayores niveles de competencia en el sistema educativo, mayores problemas en el mundo laboral, etc. Esto último repercutirá entre otras cosas sobre sus posibilidades de encontrar un empleo, sobre su salario y sobre sus posibilidades de ascenso en la escala profesional, lo que les llevará a posponer la formación de matrimonios y/o a aplazar el nacimiento de sus hijos.

El status económico relativo puede ser ahora medido a través del número relativo de jóvenes, es decir, de la proporción de individuos con edades de entrar y posicionarse en el mercado de trabajo y formar una familia (varones entre 16 y 29 años) y el número de posiciones ya consolidadas en el mercado laboral (varones entre 30 y 64 años). El nuevo indicador a manejar constituye una medida de la escasez relativa de jóvenes (ERJ) y se puede definir de la siguiente manera:

$$
\text { ERJ }=\frac{\text { Población de } 16 \text { a } 29 \text { años }}{\text { Población de } 30 \text { a } 64 \text { años }}
$$

Easterlin (Easterlin, Wachter y Wachter, 1978a) da un paso más adelante en esta idea y relaciona la estructura de edad en cada momento con el volumen de nacimientos de la generación anterior. Una variación en la tasa de natalidad de un período genera con el tiempo una variación en la estructura por edad de la población, afectando a la composición de la población en edad de trabajar y con ello a las posiciones económicas relativas de los individuos. En última instancia los efectos van a repercutir en la fecundidad de esa generación. En otras palabras, la tasa de fecundidad actual depende de la estructura por edad actual; la estructura por edad actual depende de la fecundidad de años anteriores y, consecuentemente, la fecundidad actual es el producto de la fecundidad pasada. A partir de ahora la hipótesis de Easterlin implica un mecanismo de onda a largo plazo que se autorreproduce gracias al efecto del número relativo de jóvenes en la población activa sobre sus ingresos relativos. Estamos ahora ante unas fluctuaciones demográficas que son autodependientes, pues la fecundidad de una generación es función en última instancia de la fecundidad de la generación anterior.

\section{El análisis de la evidencia: \\ los intentos de verificación de la hipótesis de Easterlin}

Son muchos los trabajos de índole empírica y teóricos desarrollados a partir de la obra de Easterlin por demográfos, sociólogos, economistas, historiadores, etc. Heterogeneidad es la palabra que mejor define toda esta literatura generada en los últimos años: los distintos trabajos difieren considerablemente en las metodologías empleadas, en los marcos temporales de referencia, en las variables consideradas, etc. Se procederá a continuación a revisar algunos de los más importantes trabajos empíricos desarrollados con el objeto de poner 


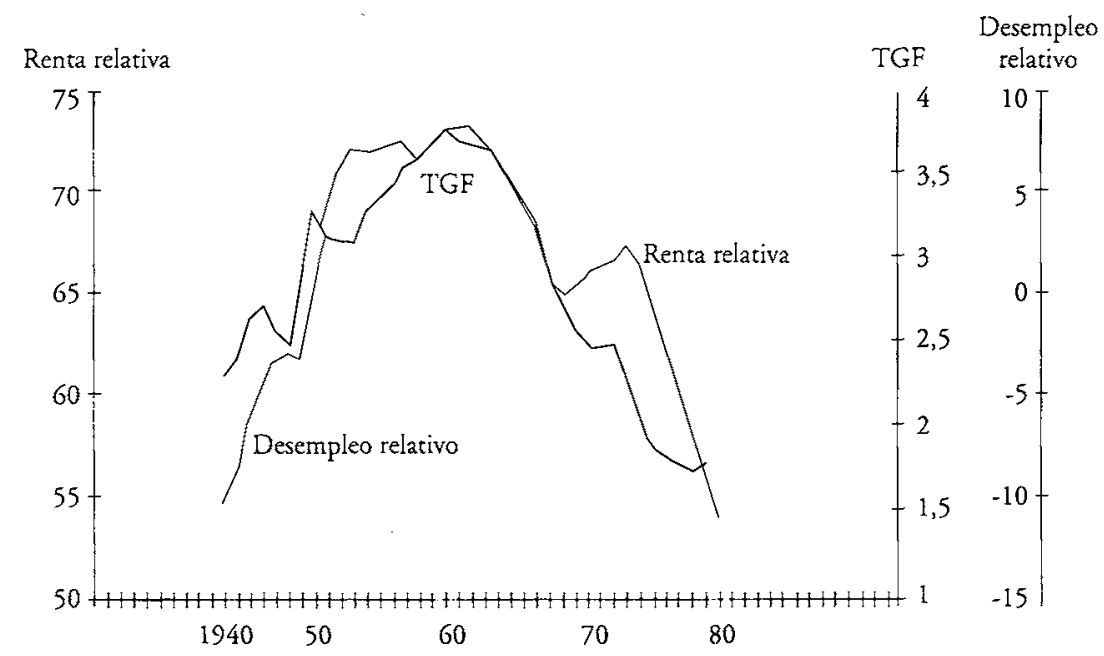

Gráfico 1. Fuente: Easterlin (1987a), p. 50.

a prueba la hipótesis de la renta relativa ${ }^{17}$. En primer lugar se analizará el grado de ajuste de la teoría al caso norteamericano. A continuación se revisarán los resultados obtenidos para otros países, sefialando finalmente los distintos trabajos realizados para el caso español.

El propio Easterlin proporciona junto a su teoría evidencia empírica de que su modelo se ajusta a la evolución de los hechos en el caso de los Estados Unidos. Los procedimientos técnicos que utiliza para ello son inicialmente muy simples, lo que desencadenó en su momento algunas críticas acerca de la escasez de formalización y sofisticación en el empleo de técnicas estadísticas (Kelley, 1972). Easterlin pone en práctica básicamente dos tipos de test. En el primer caso (Easterlin, 1962, 1965, 1966, 1968a y 1973) Easterlin analiza el efecto de las variaciones en la situación económica de los individuos sobre el número de hijos de éstos. Se trata aquí de poner a prueba la idea de que a medida que aumenta el status económico relativo de las parejas jóvenes, aumenta el número de hijos de éstas.

La relación inicial se ve confirmada con los datos. Los resultados para este primer test se pueden observar en el gráfico 1 , donde se recoge la evolución de la tasa de desempleo y renta relativa y la tasa global de fecundidad desde 1940 hasta 1978. La posición económica relativa de los jóvenes varones mejora entre 1940 y finales de los años cincuenta, yendo acompañada de un aumen-

17. En ningún caso se pretende efectuar un análisis exhaustivo y crítico de todos ellos, tarea que ya ha sido realizada por Rodríguez Sumaza (1994). 
to en la tasa global de fecundidad. A partir de 1960 ambas variables disminuyen conjuntamente.

Son varios los trabajos empíricos que intentan verificar la versión económica de la hipótesis de Easterlin. El primero de ellos tiene lugar en 1963 y es llevado a cabo por Freedman (1963), quien demuestra que la variable que mejor explica las variaciones en la fecundidad es la renta relativa del esposo. Desde entonces, autores como Wachter (1975), Conger y Campbell (1978), Crimmins-Gardner y Ewer (1978), Thornton (1978a) y Schapiro (1988) confirman sistemáticamente la idea de que las variaciones en la renta relativa conllevan variaciones en la fecundidad.

Otros autores se muestran abiertamente críticos ante esta idea. Así, MacDonald y Rindfuss (1978) afirman que el status económico relativo tiene escaso efecto sobre la fecundidad de las mujeres casadas menores de 45 años, si bien su estudio se limita a los tres primeros años del matrimonio. Olneck y Wolfe (1978) sostienen que las diferencias en el nivel de ingresos no explican diferencias en el número de hijos y Rutten y Higgs (1984) niegan al soporte técnico de las afirmaciones de Easterlin a partir de un análisis de los gráficos que éste último utiliza para recoger la evolución conjunta de la renta relativa y la fecundidad. El papel del concepto de aspiraciones materiales de los individuos es puesto a prueba con satisfactorios resultados por Coombs y Zumeta (1970), Lindert (1977) y Elder (1981). Un indicador posible de éste, la riqueza de la familia de origen, también muestra poseer grandes efectos negativos sobre la fecundidad de los hijos (Moffit, 1982). A juicio de otros autores no se puede afirmar, sin embargo, que el status económico relativo sea el factor más importante a la hora de explicar la fecundidad, asignando dicha responsabilidad a las variaciones de los salarios (Butz y Ward, 1979). La tesis defendida por ambos proclama la naturaleza anticíclica del comportamiento de la fecundidad, en contra de la postura de Easterlin.

Cuando Easterlin y Condran (1976) introducen la nueva variable (el número relativo de jóvenes en edad laboral o escasez relativa de jóvenes) en el esquema explicativo inicial, para el caso estadounidense se aprecia claramente cómo entre 1940 y 1970 los aumentos en la tasa global de fecundidad son posteriores y siguen en su tendencia a la evolución de la escasez relativa de jóvenes. Asimismo, cuando aumenta la proporción de jóvenes, se observa con un lag un descenso en los niveles de fecundidad. A partir de entonces (Easterlin y Condran, 1976; Easterlin, 1976a, 1978a y 1980a) se trata de ver el efecto de las variaciones en el número relativo de jóvenes sobre la fecundidad y se contrasta la idea de que a menor tamaño de las cohortes de los individuos, mejores posibilidades de éxito económico y por tanto mayor libertad para tener mayor número de hijos.

Este segundo test estaría implícito en el gráfico 2, donde se recoge la evolución conjunta para idéntico período de tiempo de la tasa global de fecundidad y el número relativo de varones jóvenes. Entre 1955 y 1960 el ratio varones entre 30 y 64 años y varones entre 15 y 29 aumenta, indicando una creciente escasez relativa de jóvenes. La fecundidad se mueve en idéntico sentido. A partir de 1960 los 


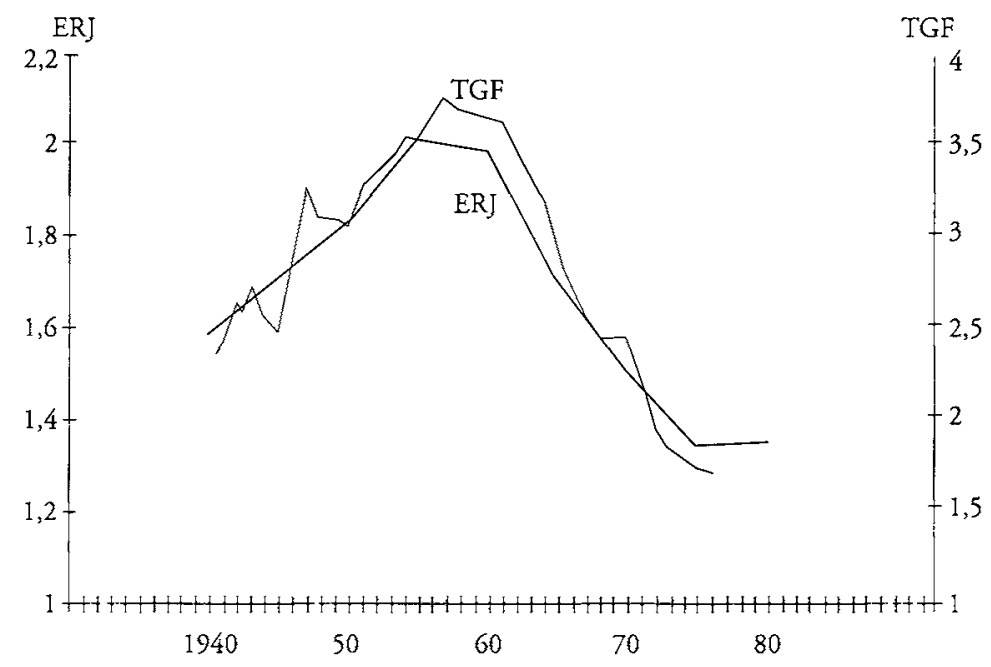

Gráfico 2. Fuente: Easterlin (1987a), p. 51.

varones jóvenes comienzan a ser cada vez más abundantes, lo que ensombrece sus posibilidades económicas y tiene su inmediato reflejo en el número de hijos.

En Easterlin, Wachter y Wachter (1978a) se hace depender el número relativo actual de jóvenes de la natalidad de las generaciones antecedentes (20 ó 25 años antes). Una vez demostrada la relación entre ambas variables, se pasa al estudio de la relación existente entre la natalidad antecedente y la natalidad actual. La conclusión final es que la fecundidad actual está condicionada por el número de nacimientos que tuvieron lugar con anterioridad, dado el efecto intermedio de éstos últimos sobre la escasez relativa de jóvenes. El estudio de estas relaciones también se puede encontrar en los artículos de Easterlin (1978a, 1978c y $1987 \mathrm{a}^{18}$ ), quedando así definitivamente diseñada la versión demográfica de la explicación a nivel agregado de las variaciones de la fecundidad de Easterlin ${ }^{19}$.

En artículos posteriores los procedimientos a que Easterlin recurre para verificar su propuesta se sofistican considerablemente. En colaboración con M.A. Artzruoni (Easterlin y Artzrouni, 1982), Easterlin estudia la evolución conjunta de las series históricas de nacimientos y fecundidad en Estados Unidos entre 1951 y 1974, bajo la idea de que la estructura por edad de un país es el producto de la trayectoria previa de los nacimientos ${ }^{20}$.

18. El artículo denotado como Easterlin (1987a) es una segunda edición actualizada del libro que con el mismo título se publicó en 1980.

19. Los gráficos 1 y 2 son considerados por Easterlin como la evidencia a favor de su hipótesis: la hipótesis de la renta relativa permite explicar el baby boom y el baby bust ocurrido en Estados Unidos.

20. Esta idea, que ha sido desarrollada ampliamente por Easterlin, también ha sido estudiada con profundidad por el coautor de este artículo (ver Artzrouni, 1981). 
Los autores desarrollan en este artículo una nueva medida de la estructura por edad de la población: la tradicional medida del número relativo de jóvenes en un momento $t$ (población entre 35-64 años / población entre 20-34) se sustituye ahora por otro ratio diferente que mide el número de nacimientos ocurridos entre 35 y 64 años antes del momento $t$ en relación con el total de nacimientos habidos entre 20 y 34 años antes de $t$. Esta nueva ratio recogería perfectamente la estructura de edad en el supuesto de que no hubiera mortalidad ni migración.

Este artículo es especialmente importante desde el punto de vista metodológico para la puesta a prueba de la hipótesis de la renta relativa. Con él Easterlin deja definitivamente claro que la variable determinante de la estructura por edad actual de la población es el número de nacimientos anteriores, y no la variable tasa de natalidad de las generaciones anteriores como se proponía en Easterlin (1978a, 1978c y 1987a). El resultado que obtienen es que efectivamente en el caso de Estados Unidos la evolución de esta ratio se ajusta a la evolución de la fecundidad, lo que confirma la idea de que la escasez relativa de jóvenes supone inevitablemente un descenso en la tasa de fecundidad.

En un artículo más reciente, Easterlin y Macunovich (1988) aplican un análisis bivariante - un test de causalidad de Granger-Sims - para analizar el efecto de la actividad económica sobre la fecundidad entre 1958 y 1984 con datos mensuales $^{21}$. Como indicador de las oscilaciones en la actividad económica se utilizan las tasas de desempleo de varones y mujeres por edad; la fecundidad se mide a través de la tasa de fecundidad de las mujeres entre 20 y 24 ańos. El resultado al que llegan es que para el caso de Estados Unidos hay una relación de causalidad simple entre el desempleo y la fecundidad ${ }^{22}$. Respecto del signo, se obtiene que en los 9 a 16 meses anteriores a los datos de fecundidad, el signo del efecto del desempleo (tanto para varones como para mujeres) sobre la fecundidad es negativo. La amplitud del lag estimado existente entre el paro y la fecundidad es de 2 a 3 meses en el período 1959-73 y de 2 meses entre 1973-84.

Por último, Easterlin, MacDonald y Macunovich (1990) estudian empíricamente las series estadísticas oficiales de renta para el período 1964-87. Los datos de ingresos reales de los varones por cohortes de pertenencia confirman que los individuos pertenecientes a cohortes más numerosas se enfrentan a peores condiciones en el mercado de trabajo. Como consecuencia de ello, una serie de cambios demográficos tienen lugar para compensar el deterioro de la situación económica, entre los que destacan la reducción en el número de hijos, la mayor participación de las esposas en el mercado de trabajo, la compaginación de las actividades domésticas con. las extradomésticas, etc ${ }^{23}$.

21. El período total se fracciona en dos subperíodos; uno de ellos va desde 1958 hasta 1973 y el segundo desde 1973 hasta 1984 , ya que se considera que en $1972-4$ hay un cambio estructural en la evolución de las variables.

22. No hay efecto feedback entre la fecundidad y el desempleo.

23. Los resultados de este trabajo están en la línea de los desarrollados por Danziger y Gottschalk (1986), Levy (1987), Levy y Michel (1986), Lillard y Macunovich (1989) y Welch (1979) (Easterlin, MacDonald y Macunovich, 1990, p. 278). 
La versión demográfica de la hipótesis de Easterlin queda validada con los trabajos de Lee (1974), Samuelson (1976), O'Connell (1978), Berger (1981, 1985 y 1989) y Baird (1987). Todos ellos constatan la importancia de la variable tamaño relativo de las generaciones a la hora de comprender las variaciones en el número final de hijos por mujer, dado su efecto negativo sobre las oportunidades económicas de los individuos. Especialmente interesantes son los estudios de Ahlburg (1982a, 1982b y 1983) sobre la naturaleza cíclica de la fecundidad. Sus trabajos demuestran cómo los niveles de natalidad de hace veinte años en Estados Unidos condicionan la estructura por edad de la población actual y ésta, a su vez, la fecundidad de las generaciones actualmente en edad reproductiva.

En el caso norteamericano la validez de la hipótesis de la renta relativa queda mayoritariamente comprobada. Los resultados no son tan claros sin embargo cuando se trata de analizar otros lugares. La relación entre el status económico relativo y la fecundidad en el caso de Canadá ha sido sometida a prueba en diversas ocasiones. Así, mientras que Chaudhury (1977), Robertson y Roy (1982) y Ahlburg (1986) confirman la hipótesis easterliniana, Wright y Maxim (1987) niegan que la tasa de fecundidad se vea afectada por la escasez relativa de jóvenes.

En Finlandia los resultados obtenidos por Sweetser y Peipponen (1967) demuestran que cambios en la composición por edad de la población condicionan el comportamiento en materia de fecundidad, ya que modifican las oportunidades del mercado de trabajo y las condiciones retribituvas de los trabajadores. Similares resultados obtiene Leridon (1978) en su estudio del caso francés y Mosk y Nakata (1985) para Japón. Ermisch (1979) difiere de las ideas de Easterlin al llegar a la conclusión de que el factor dominante en la explicación de las variaciones de la fecundidad en Gran Bretaña no es el status económico relativo, sino los distintos niveles de retribución salarial de los individuos.

También existen trabajos que proporcionan un análisis comparativo entre diferentes países. Destaca de entre ellos el realizado por Bourgeois-Pichat (1979), que afirma que en todos los países estudiados ${ }^{24}$ la fecundidad de los padres influye negativamente en la de los hijos. Para Chesnais (1983) sólo en siete países ${ }^{25}$ de los diecinueve estudiados ${ }^{26}$ el tamańo relativo de la cohorte tiene un papel decisivo a la hora de explicar las vartaciones en la fecundidad. En la mayoría de los países con cuyos datos trabaja O'Connell $(1978)^{27}$ existe una significativa relación inversa entre las variaciones en el número de jóvenes varo-

24. Francia, Estados Unidos, República Federal de Alemania, República Democrática de Alemania, Inglaterra y Gales.

25. Estados Unidos, Canadá, Australia, Francia, Bélgica, Finlandia y Países Bajos.

26. Además de los ya citados se analizan los casos de Noruega, Japón, Suecia, Suiza, Inglaterra y Gales, Dinamarca, República Federal de Alemania, Austria, Italia, España y Portugal.

27. Estos son Canadá, Estados Unidos, Inglaterra, Dinamarca, Noruega, Suecia, Australia, Nueva Zelanda, Holanda, Bélgica y Finlandia. 
nes y el índice sintético de fecundidad. Por el contrario, en tres de ellos ${ }^{28}$ la relación es positiva y en otros tres ${ }^{29}$ no existe relación.

El propio Easterlin intenta la extrapolación de sus afirmaciones a otros países, encontrando una gran similitud en la evolución de las variables estructura por edad y fecundidad en todos ellos ${ }^{30}$ (Easterlin y Condran, 1976) y un alto nivel de significación en las correlaciones entre la estructura por edad y la fecundidad para todos ${ }^{31}$, excepto para Suiza (Easterlin y Artzrouni, 1982).

Sólo Wright (1989), tras observar que no en todos los países europeos el tamaño relativo de la cohorte presenta una relación positiva de causalidad sobre la fecundidad, niega la consideración de la hipótesis de Easterlin como una explicación universal, señalando la necesidad de incorporar variables adicionales en el modelo tales como los salarios de varones y mujeres y las diferentes tasas de participación en el mercado de trabajo.

Hasta aquí se ha omitido el comentario de los distintos trabajos empíricos sobre el caso de España, cuando éste era considerado. Dicho análisis se efectua a continuación, comenzando por los estudios comparativos donde se trata a España junto a otros países, para pasar finalmente a analizar aquéllos que se ocupan en exclusiva de su singularidad. La hipótesis de Easterlin ha sido sometida a prueba en el contexto español en varios estudios con desiguales resultados. El primero de ellos es el de Easterlin y Artzrouni (1982), donde los autores comprueban la capacidad de la variable número de nacimientos para medir la estructura por edad de la población, y una vez comprobado esto, miden la relación entre esta nueva estructura por edad imputada y la fecundidad, con lo que obtienen un elevado nivel de correlación. El principal problema de este trabajo es de naturaleza metodológica, pues los autores confiesan que para el período analizado (1951-76) sólo disponían de cinco datos para España aunque, según ellos, éstos abarcaban y representaban bien a dicho período.

El segundo intento de validar la hipótesis de Easterlin en el caso español es el de Chesnais (1983), que estudia la evolución conjunta del número relativo de jóvenes y la tasa neta de reproducción para 1930-80 en dieciocho países. Los resultados para Espańa señalan la existencia de una relación "débil y sin tendencia acusada" (Chesnais, 1983, p. 381), al igual que ocurre en otros dos países de la Europa meridional. Cabría aquí señalar que el período elegido por el autor para el estudio no es adecuado en el caso español, ya que son los años de la Guerra Civil y posteriores aquéllos en los que la tasa neta de reproducción ofrece una trayectoria más accidentada, presentando las variables fecundidad y estructura por edad a partir de 1950 una mayor sincronía en su evolución. No hay que olvidar tampoco que la transición demográfica española es más tar-

28. Japón, Austria y Suiza.

29. Francia, Portugal y Hungría.

30. Canadá, Estados Unidos, Australia, Inglaterra y Gales.

31. Estados Unidos, Francia, Finlandia, Inglaterra y Gales, Italia, España, Dinamarca, Países Bajos y Suecia. 
día que en el resto de países europeos, con lo que el período a elegir ha de tener en consideración la década de retraso con que en España se realiza el tránsito a un régimen demográfico moderno.

En 1989 Wright analiza la posible existencia de una relación de causalidad entre el tamaño relativo de la cohorte y la tasa global de fecundidad a lo largo del período 1950-85. Los resultados son contradictorios según los distintos países, y es imposible para el caso de España detectar la presencia de dicho fenómeno. Para Wright la hipótesis de Easterlin no se verifica en el caso espańol puesto que la escasez relativa de jóvenes no ha aumentado en los últimos años del período estudiado. Wright parece también olvidar ese retraso con que las variables demográficas en Espafia evolucionan respecto al resto de Europa. Si para 1985, fecha última que él considera, la escasez relativa de jóvenes aún no ha comenzado a crecer, es, entre otras cosas, porque aún no ha finalizado su fase descendente.

La validez de la hipótesis de Easterlin exclusivamente para el caso español genera en la década de los ochenta un amplio debate, dados los resultados contradictorios que obtienen los distintos analistas. El primer trabajo es llevado a cabo por Agüero y Olano (1980). Ambos autores calculan la correlación entre la escasez relativa de jóvenes y la tasa bruta de natalidad y la escasez relativa de jóvenes y la tasa bruta de reproducción para el período 1960-75, en ambos casos se obtiene un coeficiente de correlación no significativo.

En 1984 Gil Calvo realiza su propia prueba de validación y concluye en base a las correlaciones efectuadas entre la escasez relativa de jóvenes y la tasa bruta de natalidad y la tasa de ocupación y la tasa bruta de natalidad para el período 1964-81, que el número relativo de jóvenes determina su situación laboral, siendo esta última variable la que condiciona la natalidad.

El principal problema que presentan ambos artículos es que la variable que eligen como más representativa para medir las decisiones en materia de fecundidad de las parejas - la tasa bruta de natalidad - no es la variable adecuada para intentar validar la hipótesis de la renta relativa. Según Easterlin, es preciso recurrir para ello a la tasa global de fecundidad, ya que sólo esta variable "refleja la influencia de los cambios en el comportamiento en materia de matrimonio y de la fecundidad dentro del matrimonio» (Easterlin, 1987a, p. 48). También hay que señalar que Easterlin y Artzrouni (1982) dejan bien claro que sólo las series históricas de nacimientos constituyen una aproximación ${ }^{32}$ a la estructura real por edad de la población actual.

En 1986 Gil Calvo publica otro artículo que, aunque tiene como objetivo analizar la evolución de la población juvenil española y el empleo de su tiempo libre, utiliza de nuevo la idea easterliniana de que el tamaño de la generación a la que se pertenece influye en las oportunidades de empleo de los individuos $^{33}$. Adicionalmente el autor proyecta hasta el horizonte 1997 el

32. Porque no se tienen en cuenta ni las migraciones ni la mortalidad.

33. Esta misma idea es desarrollada con más profundidad en el artículo de 1988 en que Gil Calvo estudia el desempleo juvenil en España y la Comunidad de Madrid. 
número de jóvenes, pero en este caso a partir del número de nacimientos antecedentes, y no en base a las tasas de natalidad antecedentes.

Gil Calvo (1989a) ${ }^{34}$ reconoce el error de su primera publicación (Gil Calvo, 1984) y con ello la necesidad de recurrir a los datos de nacimientos en vez de a las tasas de natalidad antecedentes para realizar las proyecciones ${ }^{35}$. En este trabajo el autor pasa a considerar no sólo el volumen de población masculina, sino también el total de población (varones y mujeres), centrándose a estudiar la evolución futura del volumen de jóvenes como una función del número de nacidos de 15 a 29 años antes en España y la Comunidad de Madrid.

Por primera vez en este artículo Gil Calvo, apoyándose en los trabajos desarrollados por Mathilda Riley (1972, 1979, 1981 y 1988) en el ámbito de la Sociología de la Edad, propone analizar el ciclo demográfico como una función exclusivamente del tamaño de las cohortes femeninas. A mayor tamaño de las cohortes de mujeres jóvenes, mayor retraso en la formación de familias y mayor aplazamiento de los nacimientos.

Esta idea es desarrollada con más detalle en Gil Calvo (1989b y 1990), donde se explica la mayor participación en el mercado de trabajo de las mujeres mayores de 25 años como consecuencia, entre otros factores ${ }^{36}$, del tamaño relativo de las generaciones a las que éstas pertenecen: cuando las cohortes son poco numerosas (por ejemplo entre 1971 y 1977), disminuye la participación en el mercado de trabajo (por ejemplo entre 1973 y 1976); si las cohortes aumentan de tamańo (como entre 1965 y 1971 y a partir de 1977), la «retirada del empleo disminuye». Dada la relación entre el tamaño de las cohortes femeninas y la presencia de la mujer en el mercado de trabajo, Gil Calvo (1989b) se pregunta si es lícito relacionar el tamańo de las cohortes femeninas y la fecundidad dada la intermedia relación negativa entre la participación de la mujer en el mercado de trabajo y la fecundidad.

$\mathrm{Su}$ pregunta supone un interrogante que, aunque se apoya inicialmente en la misma idea acerca del papel decisivo del tamaño de las cohortes en la vida de los individuos que utiliza Easterlin, difiere radicalmente en varios sentidos. En primer lugar, para Easterlin nunca fue el tamaño de las cohortes progenitoras la variable clave, sino el tamaño relativo de éstas en relación con el número de adultos. Por otra parte, Easterlin nunca creyó en la existencia de una relación de causalidad entre la participación femenina en el mercado laboral y la natalidad, sino que considera a ambas el resultado de la evolución en la situación

34. El objetivo de este artículo, al igual que ocurre con Gil Calvo (1986), no es poner a prueba la validez de la teoría de Easterlin. Dicho artículo se centra en el estudio comparativo de la relación adultos/jóvenes en el total nacional y para la Comunidad de Madrid.

35. Si para obtener la cantidad de jóvenes en un año partimos de la tasa de natalidad antecedente, entonces obtenemos cantidades relativas de jóvenes. Si por el contratio partimos de número de nacimientos ocurridos entre 15 y 29 años antes, entonces conseguimos cantidades absolutas de jóvenes. Este último es el dato que necesitamos para construir la escasez relativa de jóvenes propuesta por Easterlin.

36. También la coyuntura histórica y económica y la escolaridad explican parte de esta nueva tendencia. 
relativa de las parejas. Y tercero, Easterlin nunca recurre a la medición del tamaño relativo de las cohortes femeninas, ya que considera que las decisiones de formación de la familia se toman básicamente en función de la situación de los varones. La hipótesis avanzada por Gil Calvo resulta, no obstante, extremadamente interesante y constituye sin duda un interesante campo de trabajo adicional a investigar.

En otro artículo Gil Calvo (1989b) concluye recurriendo a la tradicional propuesta de Easterlin. Se analiza aquí además de España el caso de varios países europeos (Dinamarca, Holanda, Alemania e Italia), para los que se comprueba un rebrote en la proporción de matrimonios y en la fecundidad inmediatamente posteriores a las reducciones en el número relativo de jóvenes. Se predice para España el mismo efecto, pues en 1987 se alcanzó aquí el máximo en la proporción relativa de jóvenes (varones entre 15 y 29 años I varones entre 30 y 64 años).

Finalmente, en Rodríguez Sumaza (1994) se procede a una contrastación de la hipótesis en su versión demográfica con el objetivo de comprobar si efectivamente, tal y como Easterlin sugiere, la escasez relativa de jóvenes - considerada como un indicador del status económico relativo- tiene un efecto causal sobre la fecundidad. Para estudiar la posible existencia de causalidad entre las variables citadas se aplica el test de causalidad de Granger, empleado ya con anterioridad en Easterlin y Macunovich (1988) y Wright (1989), y se manejan datos para el período 1950-91. La hipótesis no es estadísticamente rechazada y en base a ella se procede a utilizar esa causalidad para predecir valores futuros de la tasa global de fecundidad, proyección que tiene como horizonte el año 2005.

\section{Conclusiones}

El presente trabajo ha pretendido ofrecer una visión panorámica de la aportación a la teoría demográfica de Richard A. Easterlin, en particular de su análisis de los ciclos demográficos. Se parte para ello de la idea de que la obra del citado autor constituye una interesante y fecunda síntesis de dos enfoques que hasta entonces constituían visiones alternativas: los puntos de vista sociológico y económico en relación con la fecundidad.

El estudio de la prolija y extensa obra de Easterlin pone en evidencia algo no claramente seńalado por él mismo pero que confiere a su trabajo una doble dimensión: la existencia de dos niveles diferentes en su explicación. Mientras que en determinados artículos el autor desarrollaba un modelo sistemático explicativo de las decisiones individuales de las familias sobre el número de hijos, en otros trabajos el autor explicaba a nivel agregado la evolución en el tiempo de los niveles de fecundidad, centrándose especialmente para ello en el caso de la sociedad norteamericana. Una vez clarificados los dos niveles de análisis se opta por el estudio específico del segundo de ellos, que ha trascendido con la denominación de hipótesis de la renta relativa o hipótesis de Easterlin. 
La controversia acerca de la validez de las relaciones entre las variables económicas y demográficas defendida por Easterlin ha dado origen a una extensa literatura. Si algo hay evidente a la vista de tanta bibliografía en torno a su obra es que su formulación no ha pasado desapercibida ni para economistas, ni para sociólogos, ni para demógrafos. Se puede estar o no de acuerdo con su lógica o con sus hipótesis, pero en ningún caso la lectura atenta de sus argumentos despierta indiferencia.

Independientemente del grado de aceptación personal por parte de cada uno de los diferentes autores de la explicación proporcionada por Easterlin, se aprecia un consenso general a la hora de reconocer la importancia científica de su formulación. En este sentido, nadie pone en duda que el trabajo de Easterlin ha proporcionado una importante base a partir de la cual «modelizar más adecuadamente la relación entre las variables no económicas y la fecundidad ${ }^{37}$ " (Bagozzi y Van Loo, 1978, p. 210), y «desarrollar métodos de predicción demográfica ${ }^{38}$ y modelos matemáticos dinámicos para estudiar las consecuencias de la relación entre fecundidad y estructura por edad" (Ermisch, 1979, p. 39).

$\mathrm{El}$ "esquema easterliniano" ${ }^{39}$ ha sufrido igualmente diferentes críticas, siendo una de las más comunes la ya comentada y relativa a la escasa formalización de sus hipótesis explicativas (por ejemplo: Kelley, 1972, p. 227; Bagozzi y Van Loo, 1978, p. 210). Efectivamente, la explicación que Easterlin ofrece del porqué de las variaciones en el tiempo de la fecundidad no viene acompañada desde un principio de un modelo formal claro y específico que pueda ponerse inequívocamente a prueba. Este hecho explica en parte una cuestión claramente evidente tras el repaso minucioso de los diferentes trabajos empíricos: su heterogeneidad. No existe una metodología común, cada autor selecciona un período de tiempo diferente en el que realizar su estudio y no existe consenso en la definición de los indicadores que se deben emplear a la hora de medir las variables. Algunos autores (Crimmins-Gardner y Ewer, 1978; MacDonald y Rindfuss, 1978; Devaney, 1983) llegan a introducir índices psicológicos como la valoración subjetiva de la renta propia o la anticipación de las condiciones económicas futuras, dada la supuesta relación entre la fecundidad y la situación económica relativa. Asimismo, es posible encontrar trabajos ocupados en poner a prueba la versión económica de la formulación de Easterlin (Thornton, 1978a; MacDonald, 1978), mientras que otros están más interesados en su posterior versión demográfica (Leridon, 1978; O'Connell, 1978).

Por lo que se refiere a los períodos de tiempo estudiados por los diferentes autores, casi nadie justifica la longitud de la serie que utiliza, a pesar de que éste es un factor realmente decisivo para los resultados (Chesnais, 1983, p. 368). Prácticamente el período se hace depender única y exclusivamente de la dis-

37. Variaciones en las variables no económicas afectan a las preferencias y éstas a la fecundidad.

38. Para un resumen de las diferentes posibilidades metodológicas para la predicción, ver Lee (1976).

39. Concepto introducido originariamente por Chesnais (1983, p. 373). 
ponibilidad de datos con los que trabajar. En el caso de Easterlin, como él mismo señala en repetidas ocasiones, las series utilizadas para Estados Unidos toman como fecha de partida 1940, dado el corte histórico y económico que supone la Segunda Guerra Mundial.

Para el caso de Estados Unidos se puede decir que, en general, los análisis con series de tiempo relativos al período posterior a la Segunda Guerra Mundial confirman el importante papel que los factores económicos juegan en las fluctuaciones de la fecundidad. Entre los estudios empíricos que más claramente constatan la relación entre las series cronológicas definida por Easterlin, destacan los de Lee (1974 y 1976), Wachter (1975), Easterlin y Condran (1976), Lindert (1977), Ahlburg (1982a, 1982b, 1983, 1984) y Baird (1987). También a nivel agregado se comprueba para la mayoría de los casos la relación entre tamaño de la población y estructura por edad y el status económico relativo de los individuos (por ejemplo: Anderson, 1974; Lee, 1975; Winsborough, 1975; etc.).

Sin embargo, la hipótesis de la existencia de una relación positiva entre la renta y la fecundidad actual no siempre ha sido confirmada con datos de corte transversal, como muestran los trabajos de MacDonald y Rindfuss (1978); Olneck y Wolfe (1978) o Crimmins-Gardner y Ewer (1978). Una posible justificación que el propio Easterlin (1969) da de esto es que a la hora de explicar las diferencias en el comportamiento en materia de fecundidad para los individuos en un mismo período de tiempo intervienen otros muchos factores, como por ejemplo la religión, que tienen un papel importante en la formación de preferencias y que a largo plazo son constantes.

Por lo que se refiere a los trabajos sobre la validez de la hipótesis de Easterlin en otros países, tal y como Rutten y Higgs (1984) y Baird (1987, p. 58-59) señalan, una de las notas más significativas es que en la mayoría de los casos se aplica únicamente análisis gráfico o de correlación univariante ${ }^{40}$. Los países en los que se ha intentado aplicar la hipótesis de Easterlin son principalmente países desarrollados, europeos y/o de cultura anglosajona. La elección de los países a analizar es función efectivamente de los intereses personales del autor, pero también de la disponibilidad y el acceso a las estadísticas. Los mejores resultados parecen concentrarse en aquellos países más desarrollados y más similares a Estados Unidos. Así, existen pocas dudas acerca de la capacidad de la hipótesis de Easterlin de explicar las variaciones de la fecundidad en Canadá y Australia, mientras que ya no está tan clara su validez como una explicación de tipo general para explicar la experiencia global europea (en este contexto, países muy próximos geográficamente presentan importantes diferencias en el calendario y en la amplitud de las oscilaciones de las variables).

Sería redundante insistir a estas alturas en la relevancia, tanto teórica como práctica, que ha tenido la obra de Easterlin. Por exigencias de concreción y por limitaciones de extensión no ha sido no obstante posible detenerse a comen-

40. También Kelley (1972) echa de menos la aplicación de técnicas de análisis multivariante, en general, y de análisis de la varianza, en particular. 
tar algunos de los problemas conceptuales que presenta la obra del autor, así como a indicar la gran versatilidad de muchos de sus argumentos y algunos de los posibles campos de aplicación de su teoría. Baste lo hasta aquí comentado para insistir en la importante contribución del autor al esclarecimiento de las interrelaciones causales entre las distintas variables explicativas y la fecundidad.

\section{Bibliografía}

ABRAMOVITZ, M. (1964). Evidences of long swings in aggregate construction since the Civil War. Nueva York: NBER.

AGÜERO, Isabel; OLANO, Alberto (1980). «La evolución reciente de la fecundidad en Españan. Revista Española de Investigaciones Sociológicas, núm. 10, p. 121-150.

AHLBURG, Dennis A. (1982a). Alternative approaches to forecasting US fertility. Unpublished Ph.D. Dissertation, University of Pennsylvania.

- (1982b). "The new Kuznets cycle: a test of the Easterlin-Wachter-Wachter hypothesis". Research in Population Economics, 4, p. 93-115.

-(1983). "Good times, bad times: a study of the future path of US fertility". Social Biology, 30, p. 17-23.

- (1984). "Commodity aspirations in Easterlin's relative income theory of fertility". Social Biology, vol. 31, otoño-invierno, núm. 3-4, p. 201-207.

-(1986). "A relative cohort size forecasting model of Canadian total live births". Social Biology, 33, p. 51-56.

ANDERSON, J. (1982). "An economic-demographic model of the United States labor market". Research in Population Economics, 4, p. 117-153.

ARTZRUONI, M.A. (1981). "Les processus itératifs en dynamique des populations et de la théorie d'Easterlin". Mathematiques et Sciences Humaines, 76, p. 33-46.

BAGOZZI, R.P.; VAN LOO, M.F. (1978). "Fertility as a consumption: theories from the behavioral sciences». Journal of Consumer Research, 4 (4), marzo, p. 199-228.

BAIRD, A.J. (1987). "A note on the Easterlin model of fertility in Northwestern Europe and the United States: 1950-1981\%. International Journal of Comparative Sociology, 28, p. 57-68.

BECKER, Gary S. (1960). "An economic analysis of fertility". En Demographic and economic change in developed countries. Universities-National Bureau Comittee for Economic Research, Conference Series 11, Princeton University Press, p. 201-231.

BERELSON, Bernard (1972). "The value of children: a taxonomical essay». En Population Council Annual Report. Nueva York: Population Council, p. 19-27.

BERGER, Mark C. (1981). The effect of the baby boom on the earnings growth of young males. Cambridge, MA: NBER.

-(1985). "The effect of cohort size on earnings: a reexamination of the evidence». Journal of Political Economy, junio, 3, p. 561-573.

-(1989). "Demography cycles, cohort size and earnings». Demography, 26, vol. 2, p. 311-322.

BLAKE, Judith (1968). "Are babies consumer durables? A critique of the economic theory of reproductive motivation». Population Studies, 22, p. 5-25.

BONGAARTS, John (1978). "A framework for analyzing the proximate determinants of fertility». Population and Development Review, 4 (1), marzo, p. 105-132.

BOURGEOIS-PICHAT, Jean (1967). «Social and biological determinants of human fer- 
tility in non-industrial societies». Proceedings of the American Philosophical Society, 111 (3), p. 160-163.

-(1979). «La baisse actuelle de la fécondité en Europe, s’inscrit-elle dans le modéle de la transition démographique?). Population, núm. 2, marzo-abril, p. 267-306.

BUTZ, W.B.; WARD, M.P. (1979). "The emergence of counterciclical US fertility». American Economic Review, 69, p. 318-238.

CARRASCO, Cristina (1988). «Los análisis microeconómicos de la fecundidad y de la participación de la mujer: un comentario». Cuadernos de Economía, vol. 16, núm. 47, septiembre-diciembre, p. 389-404.

CHAUdHURY, R.H. (1977). "Relative income and fertility». Demography, 14, p. 179195.

CHESNAIS, J.C. (1983). «La notion de cycle en démographie. La fécondité post transitionnelle est-elle cyclique?». Population, marzo-abril.

CONGER, D.J.; CAMPBELL Jr., J.M. (1978). «Simultaneity in the birth rate equation: the effects of education, labor force participation, income and health». Econometrica, 46, p. 631-641.

COOMBS, L.C.; ZUMETA, Z. (1970). «Correlates of marital dissolution in a prospective fertility study: a research note». Social Problems, 18 (1), verano, p. 92-101.

CRIMMINS-GARDNER, E.; EWER, P.A. (1978). «Relative status and fertility», Research in Population Economics, vol. 1, p. 247-259.

DANZIGER, S.; GOTTSCHALK, P. (1986). "Families with children have fared worst". Challenge, marzo-abril, p. 40-47.

DAVIS, Kingsley; BLAKE, Judith (1956). «Social structure and fertility: an analytic framework". Economic Development and Cultural Change, 4, p. 211-235.

Del HoYo, J.; GarCia FerReR, A. (1988). Análisis y predicción de la población española: 1910-2000. Madrid: FEDEA.

DEVANEY, B. (1983). "An analysis of variations in US fertility and female labor force participation trends». Demography, 20 (2), mayo, p. 147-161.

EASTERLIN, Richard A. (1960). "Interregional differences in per capita income, population and total income». En National Bureau of Economic Research, Trends in the American economy in the nineteenth century. Studies in Income and Wealth, núm. 24, Princeton, NJ: Princeton University Press.

-(1962). «The American baby boom in historical perspective». Nueva York, NBER Occasional Paper, 79.

-(1965). "Long swings in US demographic and economic growth: some findings on the historical pattern". Demography, vol. II, p. 490-507.

- (1966). "On the relation of economic factors to recent and projected fertility changes". Demography, 3, núm. 1, p. 131-151.

- (1968a). «Population, labor force and long swings in economic growth the American experiencem. National Bureau of Economic Research, General Series, núm. 86, Nueva York.

-(1968b). «Economic growth: an overview». En SILLS, David L. (ed.). International Encyclopedia of the Social Sciences, 4, p. 395-408. Nueva York: MacMillan.

- (1969). "Towards a socio economic theory of fertility: survey of recent research on economic factors in American Fertility». En BEHRMAN, S.J.; CORSA, L.; FREEDMAN, R. (eds.). Fertility and family planning: a world view. Ann Arbor: University of Michigan Press, p. 127-156.

-(1970a). «Population». En CHAmBerL.AIN, Neil (ed.). Contemporary economic issues. Homewood: Richard D. Irwin Inc., p. 241-272 (3a edición). 
-(1970b). An approach to fertility analysis for $L D C$ ', Paper prepared for June 29-July 3, 1970, Meeting of the United Nations Ad Hoc Committee of Experts on Programs in Demographic Aspects of Economic Development. Nueva York: United Nations.

- (1971a). "Does human fertlity adjust to the environment?». American Economic Review, 61, núm. 2, mayo, p. 399-407.

- (1971b). «The economics and sociology of fertility: a synthesis». En TiLly, Charles (ed.). Historical studies of changing fertility. Princeton: Princeton University Press, p. 57-133.

-(1973). "Relative economic status and the American fertility swing». En Family economic behavior: problems and prospects. SHELDON, E.B. (ed). Toronto: Lippincott. -(1974). "Does economic growth improve human lot?». En Nations and households in economic growth: essays in honor of Moses Abramovitz. DAVID, Paul A.; REDER, Melvin W. (ed.). Nueva York: Academic Press.

-(1975a). "An economic framework for fertility analysis". Studies in Family Planning, 6 (3), marzo, p. 54-63.

- (1975b). «Farm production and income in old and new areas at Mid-Century». En Vedder, Richard K.; KuINGAMAN, David C. (eds.). Essays in Nineteenth Century Economic History. Athens: Ohio University Press.

-(1976a). "The conflict between aspirations and resources". Population and Development Review, septiembre, p. 417-426.

- (1976b). «Population change and farm settlement in the Northern United States». Journal of Economic History, 36, 1, p. 45-75.

-(1978a). "What will 1984 be like? Socioeconomic implications of recents twists in age structure». Demography, 15, 4, p. 397-421.

- (1978b). «Fertility and female labour force participation in the US: recent changes and future prospects". En INTERNATIONAL UNION FOR THE SCIENTIFIC STUDY OF POPULATION (ed.). Economic and demographic change: issues for the 1980's. Proceedings of the Conference. Helsinki 1978 Lieja (Bélgica), vol. 3, p. 71-86.

- (1978c). "New directions for the economics of fertility». En YINGER, J.M.; CUTLER, S.J. (eds.). Major social Issues. A multidisciplinary view. Nueva York: The Free Press, p. 310-317.

-(1980a). «American population since 1940". En Martin FELISSTEIN (ed.). The American economy in transition. Chicago: University of Chicago Press.

-(ed.) (1980b). Population and economic change in developing countries. Chicago: University of Chicago Press.

- (1984). «Reply to Rutten and Higgs». Research in Population Economics, vol. 5, p. 213-215.

- (1986). «Economic preconceptions and demographic research: a comment». Population and Development Review, 12, p. 517-528.

- (1987a). Birth and fortune: the impact of numbers on personal welfare. Nueva York: Basic Books (2nd edition).

- (1987b). «Toward the cumulation of demographic knowledge». Sociological Forum, 2, 4, otoño, p. 835-842.

-(1987c). «Easterlin hypothesis». En Eatwell, J.; Milgate, M.; Newiman, P. (eds.). The New Palgrave, vol. 2, p. 1-4. Londres: MacMillan.

EASTERLIN, R.A.; ARTZROUNI, M.A. (1982). «Birth history, age structure and postWorld War II fertility in ten developed countries: an explanatory empirical analysis». Genus, 38 (3 y 4), p. 81-99.

EASTERLIN, R.A.; CONDRAN, G.A. (1976). «A note on the recent fertility swing in 
Australia, Canada, England and Wales and the United States". En RiCHARDS, H. (ed.). Population, factor movements and economic development. Studies presented to Brinley Thomas. Cardiff: University of Wales Press, p. 140-151.

EASTERLIN, R.A.; CRIMMINS, E.M. (1981). Analysis of WFS data in a socioeconomic «synthesis» framework. Paper delivered at the Conference of the International Union for the Scientific Study of Population. Manila.

- (1982). An explanatory study of the "synthesis framework» on fertility determination with WFS data. Paper delivered at the Meetings of the Population Association of America. San Diego, 30 de abril.

-(1984). "The estimation of natural fertility: a micro approach". Social Biology, vol. 31, primavera-verano, núm. 1-2, p. 160-170.

-(1985). The Fertility Revolution: a supply-demand analysis. Chicago: University of Chicago Press.

EASTERLIN, R.A.; MaCUNOVICH, D.J. (1988). "Application of Granger-Sims causality test to monthly fertility data, 1958-1984". Journal of Population Economics, 1, p. $71-88$.

- (1990). "How parents have coped: the effect of life cycle demographic decisions on the economic status of pre-school age children". Population and Development Review, 16, p. 299-323.

EASTERLin, R.A.; SCHAPIRO, M.O. (1979). "Homicide and fertility rates in the United States: a comment». Social Biology, 26, p. 341-343.

Easterlin, R.A.; Ahlburg, D.A.; Crimmins, E.M. (1981). "The outlook for higher education: a cohort size model of enrollment of the college age population 19482000». Rev. Publ. Data Use, 9, noviembre, p. 211-227.

EASTERIIN, R.A.; CRIMMINS, E.M.; KHODAIR, I. (1985). Determinants of fertility control in Egypt, 1979-80. Washington, D.C.: The World Bank.

EASTERLIN, R.A.; MACDONALD, C.; MACUNOVICH, D.J. (1990). "How have american baby booms fared?». Journal of Population Economics, vol. 3, núm. 4.

EASTERLIN, R.A.; POLLACK, R.A.; WACHTER, M.L. (1980). "Toward a more general economic model of fertility determination: endogenous preferences and natural fertilitys. En Population and economic change in developing countries. Chicago: University of Chicago Press. Conference held in 1976, p. 81-149.

EASTERLIN, R.A.; SCHAPIRO, M.O. (1979). «Homicide and fertility rates in the United States: a comment». Social Biology, 26, p. 341-343.

EASTERLIN, R.A.; WACHTER, M.L.; WACHTER, S.M. (1977). The passing of the Kuznets cycle: is there a life after death? Presented at American Economic Association Meetings, Nueva York, diciembre.

- (1978a). «Demographic influences on economic stability: the United States experience». Population and Development Review, marzo, p. 1-22.

-(1978b). "The changing impact of population swings on the American economy". Proceedings of the American Philosophic Society, 122, p. 119-130.

-(1979). «Here comes another baby boom». Wharton Magazine, verano, p. 29-33.

ELDER, G.M. (1981). "Scarcity and prosperity in postwar childbearing: explorations from a life course perspective». Journal of Family History, 6 (4), invierno, p. 410-433.

ERMISCH, J. (1979). "The relevance of the "Easterlin Hypothesis" and the "New Home Economics" to fertility movements in Great Britain». Population Studies, marzo, p. 39-57.

FREEDMAN, Deborah (1963). "The relation of economic status to fertility». American Economic Review, 53, p. 414-426. 
-(1976). «Introduction to the Symposium on the Easterlin Hypothesis». Population and Development Review, vol. 2, núm. 3 y 4, septiembre-diciembre, p. 411-416. FREEDMAN, Ronald (1975). The sociology of human fertility: an annotated bibliography. A Population Council Books. Nueva York: Irvington.

GIL CALVo, Enrique (1984). "La tendencia futura del paro y la fecundidad». Revista Española de Investigaciones Sociologicas, núm. 27, p. 61-77.

-(1985). Los depredadores audiovisuales. Juventud urbana y cultura de masas. Madrid: Tecnos.

-(1986). «La estructura de edades y el ocio de los jóvenes: cifras españolas». Revista Española de Investigaciones Sociológicas, núm. 35, p. 179-209.

—(1988). «Empleo juvenil y estratificación por edades». Alfoz, núm. 48-49, p. 3441.

-(1989a). «El ciclo demográfico en la Comunidad de Madrid». Economia y Sociedad, núm. 2, junio, p. 23-40.

-(1989b). «Participación laboral de la mujer, natalidad y tamaño de las cohortes». Revista Española de Investigaciones Sociologicas, núm. 47, p. 137-175.

—(1990). "La bifurcación: dos escenarios posibles del futuro femenino". Madrid: Ministerio de Asuntos Sociales, Instituto de la Mujer. Serie Debate, núm. 10, p. 39-46.

Grauman, J.V. (1960). "Comment”. En INTERNATIONAL UNION FOR THE SCIENTIFIC STUDY OF POPULATION (ed.). Economic and demographic change: issues for the 1980's. Proceedings of the Conference. Helsinki 1978. Lieja (Bélgica), vol. 3, p. 275-284. JONES, Garvin W. (1982). «Fertility determinants: sociological and economic theories». International Encyclopedia of Population, vol. 2, p. 279-286.

KELLEY, Allen C. (1972). «Relative economic status and the American fertility swings. A response». En SHELDON, E.B. (ed.). Family economic behavior. Problems and prospects. Toronto: Lippincot, p. 224-227.

KUZNETS, Simon (1930). Secular movements in production and prices. Boston: Houghton Mifflin.

- (1958). "Long swings in the growth of population and in related economic variables". Proceedings of the American Philosophical Society, núm. 102, p. 25-52.

KYRIAZIS, Stella (1987). «Approches économiques de la fécondité: une analyse comparative des théories du New Home Economic et d'Easterlin». Cahiers Québécois de Démographie, vol. 16, núm. 2, octubre, p. 167-185.

LEE, Ronald D. (1974). "The formal dynamics of controlled populations and the echo, the boom and the bust». Demography, vol. 11, núm. 4, noviembre, p. 563-585.

-(1975). Fertility, age structure and income in the US, 1947 to 1974. Paper presented at Third World Congress of the Econometric Society, Toronto.

-(1976). «Demographic forecasting and the Easterlin Hypothesis». Population and Development Review, vol. 2, núm. 3 y 4, septiembre-diciembre, p. 459-468.

-(1978). «Economic aspects of age structure. Introductory statement». En International Union for the Scientific Study of Population (IUSSP), Economic and demographic change: issues for the 80's, Proceedings at the Conference, Helsinki, 1978. Lieja (Bélgica), vol. 1, p. 399-403.

- (1979). "Causes and consequences of age structure fluctuations: the Easterlin Hypothesis». En INTERNATIONAL UNION FOR THE SCIENTIFIC STUDY OF POPULATION (IUSSP). Economic and demographic change: issues for the 80', Proceedings at the Conference, Helsinki, 1978 Lieja (Bélgica), vol. 1, p. 405-418.

LEIBENSTEIN, Harvey (1957). Economic backwardness and economic growth. Nueva York: John Wiley. 
LERIDON, H. (1978). «Fécondité et structures démographiques: une hypothésis sur l'évolution de la fécondité depuis 1940». Population, 33, p. 441-447.

LEVY, F.S. (1987). Dollars and dreams: the changing American income distribution. Nueva York: Russell Sage Foundation.

LEVY, F.S.; MICHEL, R.C. (1986), "An economic bust for the baby boom". Challenge, marzo-abril, p. 33-39.

LILLARD, L.A.; MACUNOVICH, D.J. (1989). Why the baby boom cohorts haven't boomed yet: a reconsideration of cohort variables in labor market analysis. Paper presented at the Annual Meeting of the Population Association of America, Baltimore.

LINDERT, Peter (1977). «Amercan fertility patterns since the Civil War». En LEE, R.D. (ed.). Population patterns of the past. Nueva York: Academic Press, p. 229-276.

MACDONALD, Maurice M. (1981). "Earnings, relative income and family formation". Demography, vol. 18, núm. 2, mayo, p. 123-136.

MacDonald, M.M.; Rindfuss, R.R. (1978). "Relative economic status and fertility: evidence from a cross-section". Research in Population Economics, vol. 1, p. 291-307.

MiNCER, Jacob (1963). "Market prices, opportunity costs and income effects". En Measurement in economics: studies in mathematical economics and, econometrics in memory of Yehuda Grunfeld. Stanford: Stanford University Press.

Moffrt, R.A. (1982). "Postwar fertility cycles and the Easterlin hypothesis: a life cycle approach". Research in Population Economics, 4, p. 237-252.

MONTGOMERY, M.R. (1987). "A new look at the Easterlin "synthesis" framework». Demography, 24. p. 481-496.

Mosk, C.; NAKATA, Y.F. (1985). "The age-wage profile and structural change in the Japanese labor market for males, 1964-82\%. Journal of Human Resources, 20 (1), p. $100-116$.

NAMBOODIR, N. Krishnan (1972). "Some observations on the economic framework for fertility analysis». Population Studies, 26, 2, p. 185-206.

- (1978). «On fertility analysis: where sociologists, economists and biologists meet». En YINGER, J.M.; CUTLER, S.J. Major social issues. A multidisciplinary view. Nueva York: The Free Press, p. 295-309.

OLNECK, Michael R.; WOLFE, Barbara L. (1978). "A note on some evidence on the Easterlin hypothesis». Journal of Political Econorny, 86, 5, p. 953-958.

OPPENHiEIMER, V.K. (1976). "The Easterlin hypothesis: another aspect of the echo to consider». Population and Development Review, 2, septiembre-diciembre, p. 433-458.

O'CONNELl, Martin (1978). "The effect of changing age distributions on fertility: an international comparaison". Research in Population Economics, vol. 1, p. 233-245.

RIDLEY, J.C. y otros (1987). "Farm background, socioeconomic status and fertility: the two-generation hypothesis». Social Biology, 34, otoño-invierno, p. 220-233.

RIDLEY, Mathilda (1972). Aging and society: Vol. III: A Sociology of age stratification. Nueva York: Russell Sage Foundation.

-(1979). Aging from bitrh to death. Vol. 1: Interdisciplinary perspectives. Westview Press: Boulder, Colorado.

- (1981). Aging from bitrh to death. Vol. 2: Sociotemporal perspectives. Westview Press: Boulder, Colorado.

-(1988). "Sociology of age». En Neil SMELSER. Handbook of Sociology. Chicago: University of Chicago Press, p. 243-290.

RoBERTSON, M.; ROY, A.S. (1982). "Fertility, labor force participation and the relative income hypothesis: an empirical test of the Easterlin-Wachter model on the basis of 
Canadian experience». American Journal of Economics and Sociology, 41 (4), octubre, p. 339-350.

Rodríguez SumAZA, Carmen (1994). Ciclos demográficos: una perspectiva socioeconómica. Valladolid: Universidad de Valladolid, Secretariado de Publicaciones.

RutTEN, A.; HigGS, R. (1984). "Graphic tests of Easterlin's hypothesis: science or art?". Research in Population Economics, 5, p. 201-212.

SAMUELSON, Paul A. (1976). "An economist's non-linear model of self-generated fertility waves". Population Studies, 30, julio, p. 243-247.

SANDERSON, Warren C. (1976). "On two schools of the economics of fertility". Population and Development Review, vol. 2, núm. 3 y 4, septiembre-diciémbre, p. 469-477.

SCHAPIRO, M.O. (1988). "Socio-economic effects of relative income and relative cohort size". Social Science Research, 17, p. 362-383.

SCHULTZ, Teodore W. (ed.) (1973). "New economic approaches to fertility". Journal of Political Economy, 81, núm. 2, 2a parte, marzo-abril.

-(ed.) (1974). "Marriage, family human capital and fertility». Journal of Political Economy, 82, núm. 2, 2a parte, marzo-abril.

-(1974). Economics of the family: marriage, chidren and human capital. NBER: Chicago University Press.

SPENGLER (1966). "The economist and the population question». American Economic Review, 56, p. 14-24.

SwEETSER, F.L.; PEIPPONEN, P. (1967). «Postwar fertility trends and their consequences in Finland and the US". Journal of Social History, invierno, p. 101-118.

THORNTON, Arland (1978a). "The relationship between fertility and income, relative income and subjective well-being". Research in Population Economics, vol. 1, p. 261-290.

- (1978b). The influence of income and aspirations on childbearing: results from research using multiple indicators from muliple data set. Paper presented at the Third European Colloquium on Economic Psychology, Augsburg, Germany.

WACHTER, Michael L. (1975). "A time series fertility equation: the potential for a baby boom in the 1980's". International Economic Review, 16, p. 609-624.

WELCH, F. (1979). «Effects of cohort size on earnings: the baby boom babies' finantial bust". Journal of Population Economics, 1987 (5, 2a parte), p. S65-S96.

WiNsBOROUGH, H.H. (1975). «Age, period, cohort and education effects on earnings by race». En Kenneth LAND and Seymour SPILERMAN (eds.). Social indicators models. Nueva York: Russell Sage Foundation, p. 201-218.

WRIGHT, Robert E. (1989). "The Easterlin hypothesis and the European fertility rates". Population and Development Review, 15, núm. 1, marzo, p. 107-122.

WRIGHT, Robert E.; MAXIM, Paul S. (1987). "Canadian fertility trends: a further test of the Easterlin hypothesis". The Canadian Review of Sociology and Anthropology, núm. 24 (3), p. 339-357.

WRIGLEY, E.A. (1969). Population and history. Nueva York: McGraw Hill. 